\title{
Remotely Operated Pesticide Sprayer Robot in Agricultural Field
}

\author{
Shubhangi B. Londhe \\ Student ME VLSI \& Embedded \\ System, Shree Ramchandra College of \\ Engineering, Lonikand, Pune, India
}

\author{
K. Sujata \\ HOD of E\&TC Department \\ Shree Ramchandra College of \\ Engineering, Lonikand, Pune
}

\begin{abstract}
Despite the focus on industrialization, agriculture remains a dominant sector of the Indian economy both in terms of contribution to gross domestic product (GDP) as well as a source of employment to millions across the country. Agriculture plays a vital role in the Indian economy. Over 70 per cent of the rural households depend on agriculture as their principal means of livelihood. But an attack of pest in crops is one of the major problems to reduce the production growth in agriculture field. To deal with this kind of problem diagnosis of crop disease is very essential. This paper presents an engineering solution to tackle this kind of problem in which an automatic pesticide sprayer is involved to spray the pesticide to the localized area of the affected crops. This system is based on sprayer filled with pesticides. The Sprayer movement is controlled by DC motor at low velocity, up \& down direction according to plant height. The design deals with three modules image capturing, processing and automatic pesticide spraying. The proposed system can remotely operate through any electronic device like mobile, laptop etc.
\end{abstract}

\section{Keywords}

Pesticide Sprayer, Robot, Dc motor, camera, Laptop, mobile etc.

\section{INTRODUCTION}

In India agriculture is the main source of income for Indian population which include almost 60 percent of Indian population. Agriculture where farmers work in their field to cultivate different kind of crops according to climate and resources. To deal with this kind of food demand for such huge population, farmers has to use large quantity of pesticides for increasing the food production. The productivity of crop is affected by other major biological parameter such as pest, disease and this parameter can be control by human being for improving production of crop. But it is very harmful procedure for farmers when they spray pesticide, they have to take too many precautions like wearing suitable outfit, gloves and masks etc. For getting best solutions in such cases use of robots is very imminent technological solution which improves productivity and efficiency. It becomes cost effective technological solution.

The elevation in the field of robotics has widened in different applications which extends from home automation to military. Continuous monitoring of agriculture field is possible due to automatic performance of agricultural vehicle which resulted in increased investment and research; this is one of the applications of robotic in the field of machinery design [1]. Abilities of the agricultural vehicles can be categorized as guidance, detection, action and mapping. The way of navigation by the vehicle is termed as guidance, extraction of environmental features is termed as detection and execution of the assigned task is termed as action and mapping the field with its features is mapping. All four categories are independent. An automatic vehicle which is used for main or secondary agricultural task is said to be a service unit [2-4]. An intelligent master-slave system between the agricultural vehicles developed a semi-autonomous agricultural vehicle (slave) to follow a leading tractor (master) with a given lateral and longitudinal offset [2]. A Small unmanned autonomous vehicle was developed to acquire aerial hypersprectral data, low-cost, small, lightweight hyperspectral sensor system that can be loaded onto it [3].

This system works efficiently even under unstable illumination conditions. A vehicle capable of detecting obstacles on its way and adjusting its seed was developed for tree fruit orchards [4]. This paper contains the methodology which was based on the classification and clustering of registered 3D points as obstacles. Robots implemented with RTK-GPS sensors and Wi-Fi was devised which focused on the control of several robot with respect to a reference trajectory, which was computed off-line. In this work nonlinear transformations permit to achieve a total decoupled model [5]. In this system the Process of applying chemicals can be controlled by means of wireless sensor network deployed on the agriculture field [6]

\section{THE PROPOSED SYSTEM ANALYSIS}

In the proposed system robotics model provides a facility to control the movement of agriculture vehicle. The quality and quantity of agricultural products can reduce by Plant diseases which have produced an enormous post effect scenario. Early pest detection is a major issue dealt with the plantation crops. First step involves in keen and regular observation of plants. Then the diseased plants will be classified and the affected part of the plants images will be acquired using camera. These images are then subjected to pre-processing, transformation and clustering. Then, these images are given as input to the processor, and the processor will compare the images. If the image given is affected image, then an automatic pesticide sprayer is involved to spray the pesticide to the localized area in the leaf. If not, the processors will automatic discard it and the robot will move further.

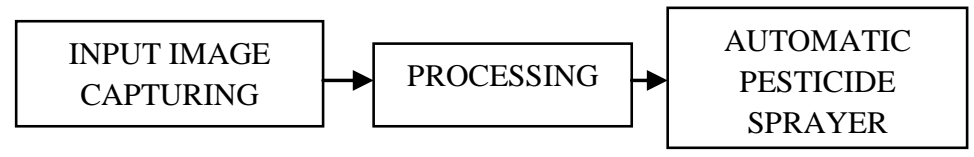

Figure 1: Basic Blocks of Pesticide sprayer Robot 
Pesticide sprayer can be used for the following purposes:

STEP1: Identifying the defective and non defective leaves in plants.

STEP2: Classifying the type of disease attacked in the leaves.

STEP3: Pesticide spraying in defective areas.

An automatic pesticide sprayer is involved to spray the pesticide to the localized area of the affected crops. This system is based on the sprayer that is filled with pesticide. This provides a continuous flow of pesticide and an accuracy that is not affected by varying fluid properties and flow conditions and also sprays pesticide on affected area of plant by adjusting the height of pesticide sprayer. This can be controlled by using Team viewer software through mobile remotely. The design is ideal for pesticide sprayer application.

\section{BLOCK DIAGRAM}

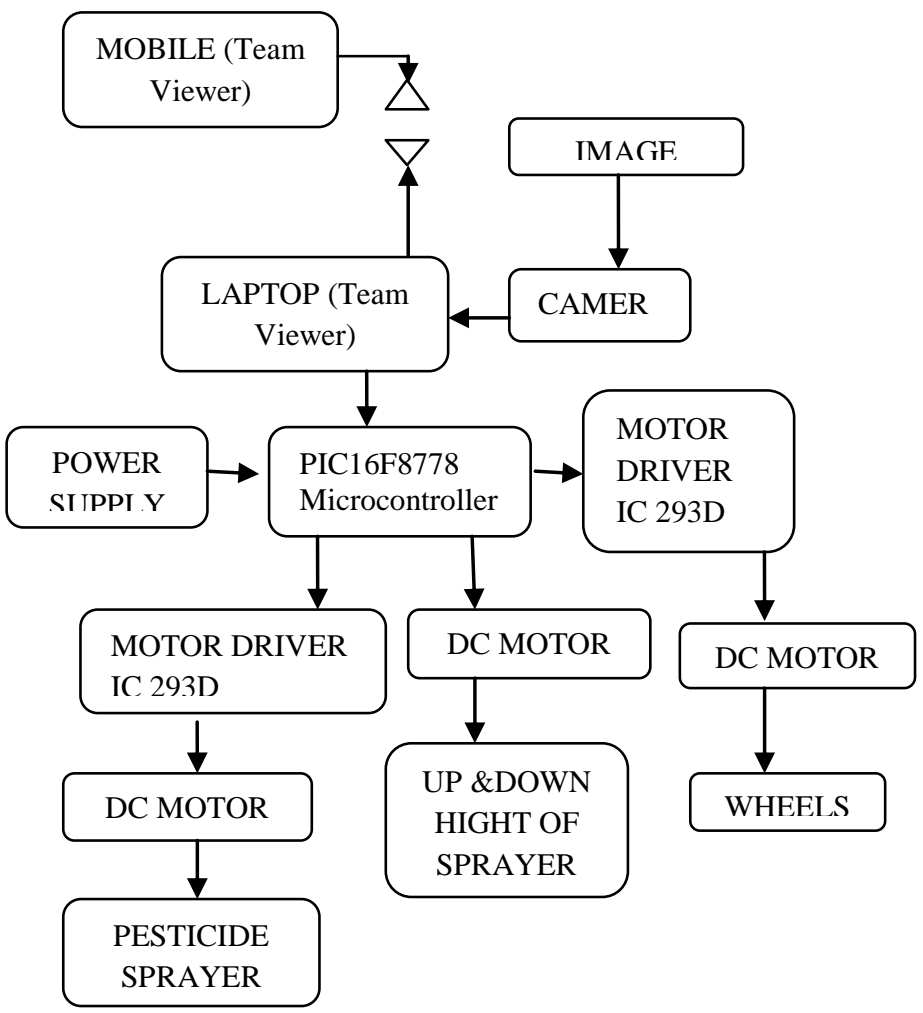

Figure 2: Block Diagram of pesticide sprayer Robot.

This system consists of three major parts:-

PART 1:- Input part

PART 2:- Spray and control processing unit

PART 3:- Output Part

Part 1: Input Part:-

- Input part consists of the power supply which provides power to each and

- Every electronic component in the Robot.

- $\quad$ LAPTOP for MATLAB using GUI.

- Camera which provides input image for Leaf disease detection.

Part 2: Spray and control processing unit.
- The PIC 16F877A Controller is the Heart of the Robot.

- The Driver Circuit is connected to the PIC Controller which in turn is connected to DC Motors for the purpose of Driving the Robot.

- The Pesticide Pump is used to transfer the pesticide from storage tank to the Sprayer head.

\section{Part 3: Output part}

- The Direction Control unit controls the direction of the robot.

- The Spraying Unit Will sprays the pesticide in specified direction.

- The spraying unit is adjusted up and down direction according to plant height.

- Whole system can be operated remotely through Mobile by using Team viewer.

Figure 2 describes the detailed diagrammatic representation of automatic pesticide sprayer. Input to the system will be the processed image; i.e. normal or defective leaf images, will be given to the PIC microcontroller. The controller will process the image and classify it as infected or normal leaf. If the input image is a pest affected image, then the processor will provide access to the relay.

The relay will act as a switch and switch on the pumping motor, which in turn spray the pesticide to the localized affected area in the infected leaves, automatically. If not, no action will be taken, the processor display it as normal image.

An automatic pesticide sprayer is involved to spray the pesticide to the localized area of the affected crops. The design is ideal for pesticide sprayer application.

\section{SCHEMATIC DIAGRAM AND DESCRIPTION}

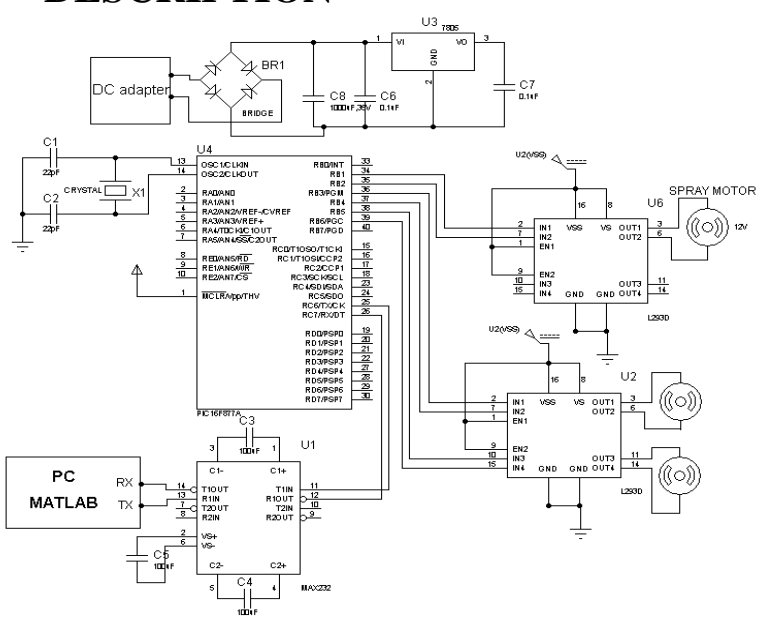

Figure 3:- Circuit diagram for Pesticide sprayer Robot

We have used PIC 16F877A Controller. Basically crystal oscillator circuit is connected to clk in and clk out pins of the microcontroller with two capacitors of $22 \mathrm{pF}$. Then, it has power supply unit which consists of bridge rectifier and filter circuit with capacitor, then by using fixed regulator IC7805, continuous $5 \mathrm{Vdc}$ supply is provided to microcontroller. Basically this circuit consists of three DC Motors one is for 
spray and another two motors are for robot. For driving these motors we have used L293d motor driver which drives these motors. Input pins of L293D drivers are connected to the microcontroller. One motor driver can drive two motors.U4 drivers one DC motor which is connected at output pins OUT1 and OUT2 of the driver IC.U3 drives two DC motors which are connected at OUT1, OUT2 and OUT3 and OUT4 pins of the driver IC. Camera is connected to the PC.PC is connected to the microcontroller through TX and RX pins.

\subsection{Motor Driver IC Interfacing}

Motor Driver IC like L293D or L298 is used for this purpose which has two H-bridge drivers. Hence, each IC can drive two motors. Motor driver does not amplify the current; it only acts as a switch (An $\mathrm{H}$ bridge is nothing but 4 switches). Drivers are enabled in pairs, with drivers 1 and 2 being enabled by the Enable pin. When an enable input is high $(\operatorname{logic} 1$ or $+5 \mathrm{~V})$, the associated drivers are enabled and their outputs are active and in phase with their inputs. When the enable pin is low, the output is neither high nor low (disconnected), irrespective of the input. Direction of the motor is controlled by asserting one of the inputs to motor to be high (logic 1) and the other to be low (logic 0 ). To move the motor in opposite direction just interchange the logic applied to the two inputs of the motors. Asserting both inputs to logic high or logic low will stop the motor.

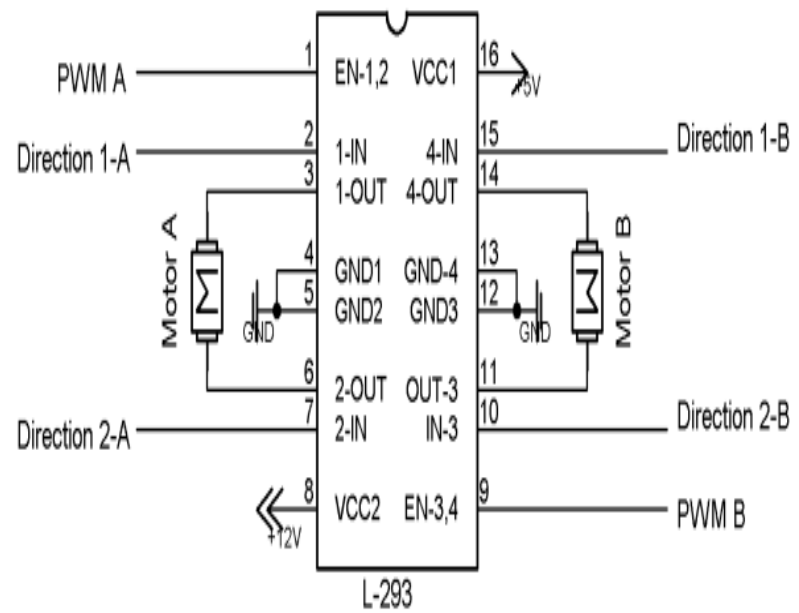

Figure 4:- L293 D Driver IC

\section{IMAGE PROCESSING}

Image processing is processing of images using mathematical operations by using any form of signal processing for which the input is an image, a series of images, or a video, such as a photograph or video frame. The basic unit of image is pixels. The group of pixels will form an image. Here in proposed system for processing an image we have used MATLAB. An image is processed for disease detection in below steps:

STEP 1:-

Firstly image has captured by camera is in the $\mathrm{YCbCr}$ form then it is converted into RGB form which shows in Figure 5.

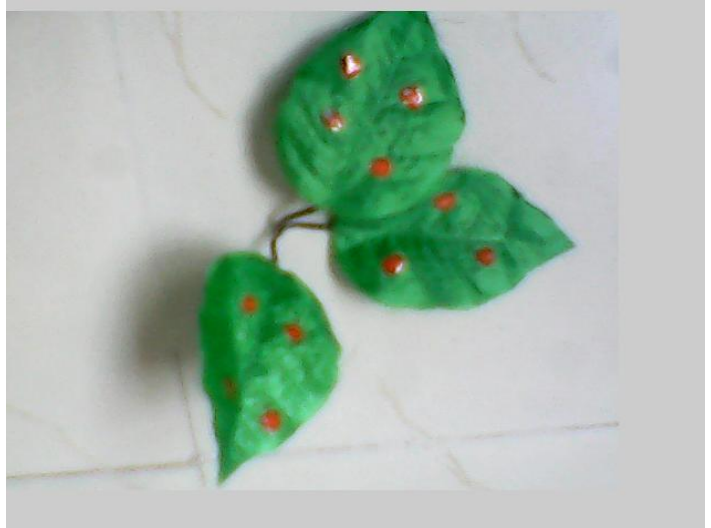

Figure 5:- Input image in RGB form.

STEP 2:-

In second step RGB image is converted into HSV image.HSV is named for 3 values: Hue, Saturation and Values. This color space describes colors (hue or tint) in terms of shade (saturation or amount of gray) and their brightness value. Which represents in Figure 6.

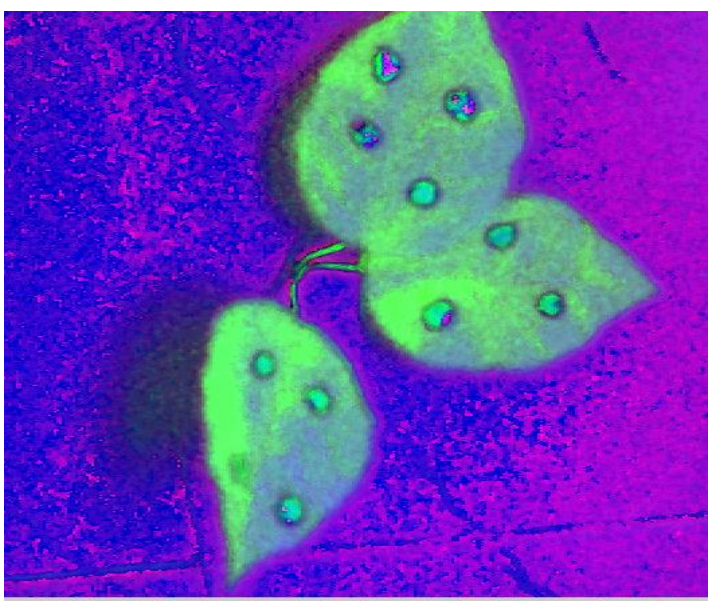

Figure 6:- HSV Image.

STEP 3:-

In step $3 \mathrm{HSV}$ image converted into Hue image as shown in Figure7.

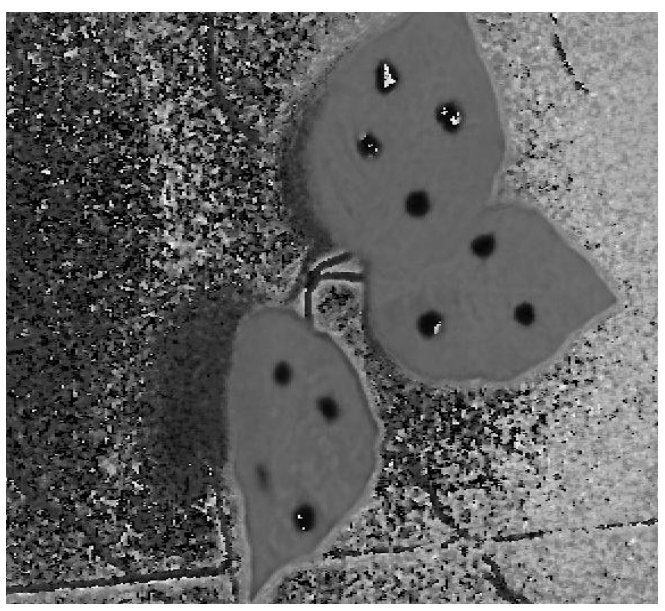

Figure 7:- Hue Image 
STEP 4:-

Figure 8 shows hue image is converted into thresholding image using MATLAB in which leaves has been segmented.

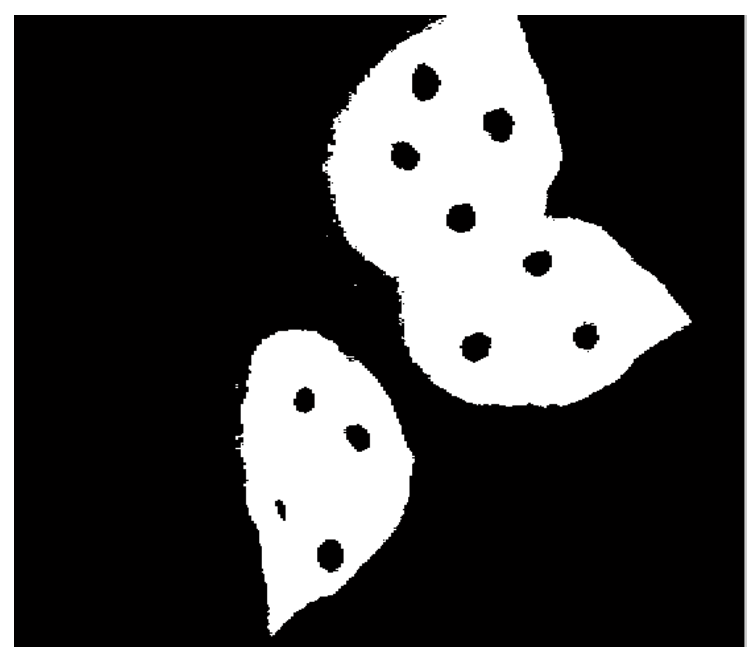

Figure 8:-Leaves segmented

STEP 5:-

In figure 9 resulted images are given in which segmented leaves image converted into noise removed image for removing another unwanted area then it is again filled with holes in filled image and when both images subtracted from each other then diseased area is resulted with Calculated percentage of diseased area .

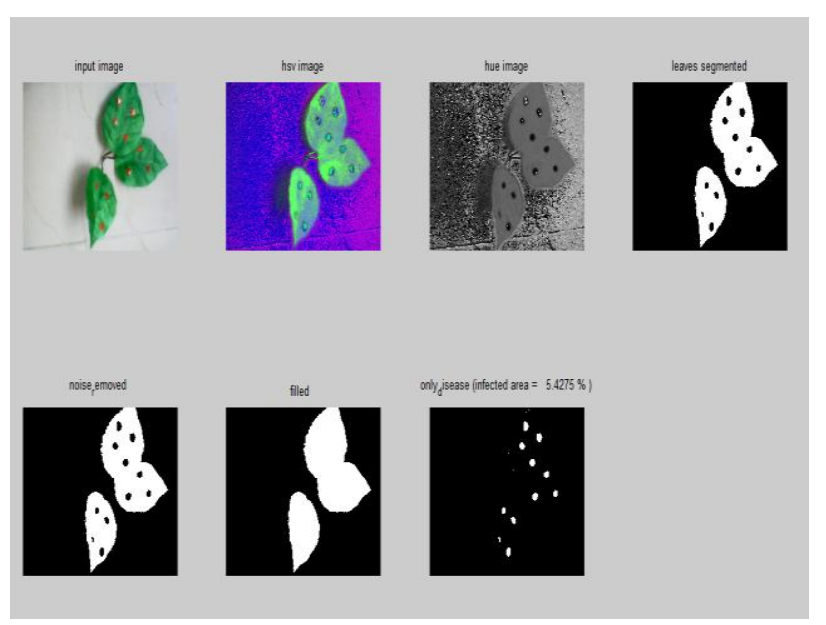

Figure 9: Resulted images.

\section{MERITS}

1. Improves the efficiency and Productivity of Agriculture

2. Replacing labourer with intelligent machine.

3. Safety to Farmers and precision is maintaining.

4. Cost effective technology

\section{FUTURE SCOPE}

1. Integrated GSM module which could control the start/stop and run operation of the robot.
2. Pre programmed GUI based navigation system.

3. Android interface to navigate the robot

4. Fertilizer drill can be incorporate for cost effective design.

\section{CONCLUSION}

The paper was aimed at not just to extend the application of advanced technology in the field of agriculture, but also to bring the technology close to the reach of farmers in financial aspect, in a very convenient way. This paper provides an engineering solution to diagnose diseased affected area of plants by automatic sprayer robot remotely. An automatic pesticide sprayer is involved to spray the pesticide to the localized area of the affected crops. This system is based on sprayer filled with pesticides. The Sprayer movement is controlled by DC motor at low velocity, up \& down direction according to plant height. The proposed system can remotely operate through any electronic device like mobile, laptop etc. This is very essential in developing countries, especially India, where agriculture is the backbone of the economy.

\section{REFERENCES}

[1] A.A.C.Fernando, and C.Ricardo, "Agricultural Robotics , Unmanned Robotic Service Units in Agricultural Tasks",IEEE Industrial Electronics Magazine, pp. 48-58, Sep 2013

[2] C.Zhang, M. Geimer, O.N.Patrick, and L.Grandl, “ Development of an Intelligent Master-Slave System Between Agricultural Vehicles", IEEE Intelligent Vehicles Symposium,pp. 250-255, San Diego, CA, US, 2010.

[3] K.Uto, H. Seki, G. Saito, and Y. Kosugi, “ Charaterization of Rice Paddies by a UAV-Mounted Miniature Hyperspectral Sensor System", IEEE Journal. Applied Earth Observations and Remote Sensing, pp. 851-860, vol.6, no.2, 2013.

[4] G. Freitas, B. Hamner, M. Bergerman, and Sanjiv Singh, A Practical Obstacle Detection System for Autonomous Orchard Vehicles, IEEE Int. Conf on Intelligent Robots and Systems, (IROS), Vilamoura (Portugal),2012.

[5] A. Guillet, R. Lenain, and B. Thuilot, Off-road Path racking of a Fleet of WMR with Adaptive and Predictive Control, IEEE Int. Conf. on Intelligent Robots and Systems, (IROS) Tokyo (Japan), 2013

[6] F.G. Costa, J. Ueyama, T. Braun, G. Pessin, F.S. Osorio, and P.A. Vargas, " The Use of Unmanned Aerial Vehicles and Wireless Sensor Network in Aggricultural Applications", IEEE International Geoscience and Remote Sensing Symposium (IGARSS), Minich, 2012.

[7] Aishwarya.B.V, Archana.G, "Agriculture Robotic Vehicle Based Pesticide Sprayer With Efficiency Optimization", IEEE International Conference on Technological Innovations in ICT for Agriculture and Rural Development (TIAR 2015), 2015. 